cerebral hemiplegia, Kallmann's syndrome, Klippel-feil syndrome, and dysplasias of the upper spinal cord. A delayed maturation of inhibitory cortical pathways is often suggested as the mechanism of mirror movements. The University College study localizes the mechanism of mirror movements in children to a delayed myelination of transcallosal inhibitory fibers, with consequent bilateral activation of cortico-spinal pathways originating from both motor cortices. Underdevelopment of the corpus callosum demonstrated by quantitative MRI in a group of children with ADHD supports the transcallosal mechanism of mirror movements in this syndrome. (see Progress in Pediatric Neurology III, PNB Publ, 1997;p212).

Precision grip and manipulation (grip-lift synergy) was examined in 13 children with spastic hemiplegia and unilateral brain lesions, in a study at the Karolinska Hospital, Stockholm, Sweden (Forssberg H et al. Brain June 1999;122:1157-1168). The developmental level of grip-lift synergy, a model of grasping and manipulation, was correlated with impaired dexterity and with extensive lesions in the contralateral cortex, white matter, thalamus, and basal ganglia. Perinatal lesions in specific cortical motor areas, not resulting in impaired dexterity, may be compensated for by other neural circuits. No specific localization was demonstrated by MRI to correlate with impaired dexterity in children with hemiplegic cerebral palsy. Instead, the memory for grip-lift synergy involves several cortical and subcortical regions.

\title{
DYSCALCULIA AND ATTENTION DEFICIT SUBTYPES
}

The association of specific academic deficits with attention deficit disorder (ADD) subtypes was determined in 20 students (ages 8-12) with ADD with hyperactivity $(\mathrm{ADD} / \mathrm{H})$ compared to 20 with $\mathrm{ADD}$ without hyperactivity $(\mathrm{ADD} / \mathrm{noH})$, at the Department of Educational Psychology, University of Texas at Austin, TX. Using subtests of the Woodcock-Johnson Psycho-Educational BatteryRevised to determine group differences, scores for students with $\mathrm{ADD} / \mathrm{noH}$ were significantly different on four of seven measures, and the Calculation subtest scores were significantly lower when compared to all other achievement subtests. By contrast, for students with $\mathrm{ADD} / \mathrm{H}$, no significant differences were found in six of seven analyses, although their Calculation subtest scores were lower than scores on the Applied Problems subtest. These results support the hypothesis that inattention has a specific and adverse effect on arithmetic computation skills. (Marshall RM, Schafer VA, O'Donnell L, Elliott J, Handwerk ML. Arithmetic disabilities and ADD subtypes: Implications for DSM-IV. L Learning Disabilities May/June 1999;32:239-247). (Respond: Richard M Marshall, Department of Educational Psychology, SZB 504, University of Texas at Austin, Austin, TX 78712).

COMMENT. Students with ADHD-Predominantly Inattentive Type are at increased risk for arithmetic calculation deficits. These findings have important implications for the diagnosis and treatment of ADHD and the recognition of ADHD subtypes as defined by the DSM-IV criteria. Children with ADD-inattentive type should also be examined for Gerstmann syndrome.

Gerstmann syndrome. Profound developmental dyscalculia and Gerstmann syndrome may occur in children with ADHD. Deficits in specific cognitive areas may involve visuo-spatial perception and parietal-occipital function. (See Millichap, Attention Deficit Hyperactivity and Learning Disorders, PNB Publ, 1998;pp82-86, for an account of Gerstmann syndrome in ADHD). Confirmation of the essential visuospatial origin and impaired mental manipulation of images is described in "a pure case of Gerstmann syndrome with a subangular lesion" (Mayer E et al. Brain June 1999;122:1107-1120). An adult 
presenting with the four characteristic symptoms (finger agnosia, agraphia, right-left disorientation and dyscalculia), in the absence of any other neuropsychological disorders, had a focal ischemic lesion in the inferior left angular gyrus, extending to the callosal fibers. An impairment in mental manipulation of images and visuospatial function is the basis for the Gerstmann cognitive deficit syndrome.

\section{PREFRONTAL DYSFUNCTION IN ADHD: FUNCTIONAL MRI STUDY}

Functional magnetic resonance imaging (MRI), during performance of two tasks requiring high-level executive control, was studied in seven adolescent boys with ADHD, and compared to nine controls, at the Institute of Psychiatry, Kings' College, London, UK. A "stop" task required inhibition of a planned motor response, and a "delay" motor timing task required the synchronization of a motor response to an intermittently appearing visual stimulus. Adolescents with ADHD showed less brain activity, predominantly in the right hemisphere mesial frontal cortex during both tasks, and in the right inferior prefrontal cortex and left caudate nucleus during the stop task. ADHD is associated with subnormal activation of the prefrontal areas responsible for higher-order, inhibitory motor control. (Rubia K, Overmeyer S, Taylor E et al. Hypofrontality in attention deficit hyperactivity disorder during higher-order motor control: a study with funcional MRI. Am I Psychiatry June 1999;156:891-896). (Reprints: Dr Rubia, MRC Child and Adolescent Psychiatry Unit, Institute of Psychiatry, De Crespigny Park, London SE5 8AF, UK).

COMMENT. Motor attention and response selection, impaired in patients with $\mathrm{ADHD}$, is associated with hypofunction of the right mesial frontal lobe and striatal areas. The findings in these functional MRI studies corroborate previous documentation by quantitative MRI of structural changes in the frontal lobe and caudate in ADHD children. (see Progress in Pediatric Neurology III, PNB Publ, 1997;p212; and Vol. II, 1994;pp172-184).

The role of the right frontal lobe in humor appreciation is evaluated at the University of Toronto and Rotman Research Institute, Baycrest Centre for Geriatric Care, Canada. (Shammi P, Stuss DT. Brain June 1999;122:657$666)$. In patients with damage to the right frontal lobe, the physical or emotional responses of laughter and smiling were diminished, and impaired performance on humor appreciation tests was correlated with cognitive deficits. Working memory, or the ability to retain information, was related to appreciation of verbal (jokes) and non-verbal (cartoon) tests.

A right frontal lobe dysfunction may explain the anhedonia characteristic of some children with ADHD.

\section{PREVALENCE OF ADHD AND METHYLPHENIDATE USAGE}

An analysis of data obtained from the National Ambulatory Medical Care Survey (NAMCS), 1990-1995, for children aged 5 through 18 years, was performed at Washington State University, to discern trends in the prevalence of US officebased visits for ADHD and for prescriptions of stimulant medications, including methylphenidate (MPH), for ADHD treatment. From approximately 1 million diagnosed cases in 1990, the prevalence of ADHD had increased 2.3-fold in 1995. In this period, the number of office visits for girls diagnosed with ADHD rose 3.9fold; the mean age of patients with ADHD increased by more than 1 year, from 9.7 to 10.8; the percentage of all office visits resulting in a diagnosis of ADHD rose from $1.1 \%$ to $2.8 \%$; and the population-adjusted rate of ADHD patients prescribed 